\title{
PROGRESSIVE DENTISTRY AND STOMATOLOGY ${ }^{1}$
}

\section{A Response to Albray's Critictsm of the Review by Stillaman and McCall, on the Etiology, Diagnosis and \\ Treatment of Periodontoclasta ${ }^{2}$}

PAUL R. STHLLMAN

New York City

AND

JOHN OPPIE MCCALL

Buffalo, New York

The criticism of our review, ${ }^{1}$ by Dr. Albray, reveals an earnest searching for truth. For this reason it demands a serious reply, irrespective of any lack of logic his argument may seem to show. For the reason, also, that his mental attitude is shared by many others who equally desire the truth, we feel impelied to make a somewhat extended reply.

Dr. Albray's remarks reveal two common characteristics of the human mind. The first is a marked tendency to retain, as accepted facts, the statements of belief regarding scientific phenomena that may be made by those whose attainments give their assertions weight. These beliefs are accepted as the truth, whether they tally with experience or not; and, by virtue of long continued acceptance, they produce in the mind of the individual a sharp reaction against contrary views. This attitude inevitably brings to mind an epigram of John Calvin's. He said: "Those who sit in judgment, pronounce for sentences the prejudices they brought from home with them." It has been said, somewhere, that educators hold to the

1 The seven preceding units in this series of reviews were published in the Journal of Dental Research by (1) Gies, i (1919), p. 525; (2) Merritt, ii (1920), p. 77; (3) Novitzky, ii, p. 567; (4) Rhein, ii, p. 579; (5) Novitzky, ii, p. 585; (6) Stillman and McCall, iii (1921), p. 73; and (7) Albray, iii, p. 339. A ninth unit, by Albray, follows and replies to this one (p. 349).-(ED.)

2 Albray: Journal of Dental Research, 1921, iii, p. 339. 
theory that it is difficult to teach a person something that he does not already know. It is a fact that unless a person has had previous contact with a subject, it is not easy for him to visualize things connected with that subject, when presented by the written word.

The subject of traumatic occlusion has been found peculiarly difficult to present in a way that will carry its full significance to him who has had no previous conscious clinical experience with it. This difficulty, together with the natural tendency to adhere to previously accepted beliefs, is at the bottom of the ideas in Dr. Albray's communication. We have found many times, in talking with dentists about traumatic occlusion, that their conception of it was quite different from the understanding of it that we were able to impart to them by actual demonstration.

We do not think that Dr. Albray would have us believe that he considers periodontoclasia a hopeless progression toward the forceps. We are very sure he has an optimistic attitude toward the hypothesis that periodontal disease, even after its inauguration, may be checked and the parts restored to health. With this as a starting point, we ask Dr. Albray and others who share his beliefs, to observe in some patient's mouth, a slightly loose upper incisor. Providing the case is not one of complete lack of occlusion in this region, we are quite confident that he will, when the patient closes his jaws in the usual position, be able to see this loose tooth moved bodily by contact with the opposing tooth. We ask Dr. Albray whether he believes it possible for this tooth to become firm and its periodontium healthy, while it is being subjected to such constant movement; and this, irrespective of the ultimate part the occlusion may have played in the etiology of the case. We then ask him to remove with a carborundum stone a sufficient amount of enamel from either the upper or lower tooth, so that the patient cannot bring these teeth into occlusal contact. We are confident that, if the tooth is free from periapical disease and has at least one half of its alveolar process intact, he will find it becoming firmer even without other treatment. Other treatment will of course be required for the complete restoration of health, but this demonstration will at least reveal the fact that traumatic occlusion plays a part in the etiology of the case.

If this phenomenon occurs, Dr. Albray will reason back to a period 
in the history of this tooth when it was still firm in its socket, but, at the same time, was being subjected to virtually the same occlusal impact. The tooth may have changed its position since it became loose, but it will be found in most cases of this kind that this migration has tended to carry the loose tooth away from the occluding tooth. In other words, the traumatic occlusion he now observes was not the product of the periodontoclasia, although it may have been exaggerated in the course of the disease.

Traumatic occlusion is difficult to detect before the affected teeth become loose. Nevertheless, by placing the finger lightly upon a suspected tooth and instructing the patient to close the jaws, a shock will be felt, even though the tooth is not perceptibly loose. Examination of such teeth will almost invariably reveal some abnormality in the gingiva or in the bone as revealed by the radiograph. Even in the cases in which the gingival abnormality is still very slight, this abnormality will be found to improve when the occlusal trauma has been relieved, an improvement of which the patient is invariably cognizant. What is to be said of the sequence of causative agencies, when the occlusal trauma is present and there is as yet no periodontoclasia?

As to the part played by diet deficient in vitamines, in the causation of periclasia, we have been unable to secure perceptible improvement by prescribing diets containing these elements. Moreover, our best advice from our medical confreres, outside the circle of those conducting animal experiments, is that vitamine-containing diets are most important in the period of childhood, becoming less significant in adult life, the period of greatest susceptibility to periclasia. Dr. Albray must pardon us if we have failed to mention this in our short review.

With regard to the various items to be considered in making a complete oral examination, we plead guilty to a misleading statement concerning our procedure. The enquiry into family and personal history, subjective symptoms, and examinations of the various organs of the body, are intended to be a corollary to and not a part of the mouth examination.

Dr. Albray wishes to know what relationship we have found between systemic disease and periodontal disease, but he himself quotes our 
answer on that subject in his succeeding paragraph. This quotation, which will be found in the second paragraph on page 83, states all that we ourselves have definite knowledge of as to the causative relationship of systemic to periodontal disease. We have drawn the conclusions there stated, as to the secondary influence of these disease conditions, because we have secured definite improvement in cases of periodontoclasia where these diseases were present, and by purely local treatment. Were these diseases primary etiologic factors in periclasia, such results could not be obtained in this manner.

Another reason, and a very important one, for enquiring into the patient's physical condition, is the determination of the possible presence of metastatic disease, since the plan of treatment may frequently be modified where such disease is present.

We fail to see why the restoration of normal approximal contacts in the adult mouth is so perplexing a problem to a practising dentist. We have never taught this subject but we know that such instruction is available. As for the supposed impracticability of orthodontic treatment for the adult: not only are cases with periodontoclasia being treated by orthodontists, but frequently, also, they cannot be restored to health by any other means. We ourselves have been impressed by the remarkable stimulation of the biogenetic forces that occurs in the periodontal tissues directly after placement of the orthodontic appliance.

With regard to the quotation on page 85 , beginning, "For as soon as the tooth surface is clean, . . . . ": we regret that the inference was drawn that correct instrumentation would entirely cure periodontal disease. What we intended to convey had reference simply to the favorable reaction that does invariably follow correct removal of effete material from the root surface. To produce this result is only a part, but a very essential part, of periodontic treatment.

We cannot help but wonder why Dr. Albray finds such a difference between our theories and their practical application, since many dentists have applied these theories in practice and have found that they do "work." Let us reiterate that periodontoclasia can be treated and cured as we have stated. It is therefore time that "every dentist" was his own periodontist. Our efforts, as exemplified in this and in other writings, are and have been directed toward this much desired consummation. 
In conclusion, we may say that many dentists have treated cases of periodontoclasia and still have found our writings convincing. It is our hope that this rejoinder may assist Dr. Albray and others to such an understanding of our views, that all who are interested may carry the investigation of periodontal disease much farther than we have been able to do. It only remains to refer him to the following papers for a fuller elucidation of etiology and the bearing of traumatic occlusion:

(a) Traumatic occlusion. Stillman: Journal of the National Dental Association, 1919, vi, p. 691.

(b) Etiology of periodontoclasia. McCall: Dental Items of Interest, 1920, xlii, p. 116.

52 Vanderbilt Avenue, New York City

360 Linwood Avenue, Buffalo, New York 